\title{
Current research topics in embodied social cognition
}

\author{
Fernando Marmolejo-Ramos • Amedeo D'Angiulli
}

Received: 10 July 2014/ Accepted: 11 July 2014/Published online: 20 July 2014

(C) Marta Olivetti Belardinelli and Springer-Verlag Berlin Heidelberg 2014

\begin{abstract}
Although a great deal of research has focused on the study of the embodiment of cognition, only recently has the association between the embodiment of cognition and social cognition been acknowledged. Current behavioural and neuroscientific research in cognitive psychology has started to provide empirical evidence, demonstrating the clear link between embodied cognition and cognitive processes dependent on social situations (see Leung et al. 2011). That is, sensorimotor experience with the environment can be shown to shape the acquisition and use of knowledge where this environment is embedded in social situations. Hence, cognition is both embodied and social and is referred to as embodied social cognition (EmSoCo). The five empirical articles composing this special section provide some examples of the cutting edge research being carried out in relation to EmSoCo.
\end{abstract}

As gestures are kinesic components essential to communication (see Cevasco and Marmolejo-Ramos 2013), their role in cognition is, therefore, straightforward. Parzuchowski et al. demonstrate that gestures of honesty, e.g. putting a hand on one's heart, can influence the judgment of others and one's actions. This supports the idea that abstract concepts are associated with concrete actions and

This editorial is part of the Special Section on 'Embodied Social Cognition', guest-edited by Fernando Marmolejo-Ramos and Amedeo D'Angiulli.

F. Marmolejo-Ramos ( $₫)$

School of Psychology, The University of Adelaide, Adelaide,

Australia

e-mail: fernando.marmolejoramos@adelaide.edu.au

A. D'Angiulli

Department of Neuroscience, Carleton University, Ottawa, Canada that non-verbal behaviour serves to convey social signals (see also Tagai et al. 2013).

That bodily actions portray and communicate social clues, requires also that emotional correlates be appraised. As shown by Stins et al., body sway is influenced by emotionally valenced facial expressions only when an explicit emotional assessment is required; when emotional assessment is irrelevant the effect vanishes. This finding demonstrates that people tend to approach positive and avoid negative situations (see Eerland et al. 2012), but adds the cautionary note that such affect only manifests when an explicit emotion is task relevant.

Marmolejo-Ramos et al. have also demonstrated that only when explicit evaluation of the emotionality of stimuli is required, does an association between emotions and concrete concepts emerge. Specifically, these researchers found that emotion concepts can be mapped on vertical spatial locations such that positive concepts are mapped onto upper locations and negative concepts onto lower locations. Although current research has already demonstrated such an association (e.g. Ansorge and Bohner 2013), Marmolejo-Ramos et al. extend those results by showing that the effect also holds for linguistic units larger than words, but only when the emotional evaluation is task relevant.

Abstract concepts, e.g. happiness and honesty, can be understood as being mediated by knowledge of concrete entities that can be linked to the abstract referents by conceptual metaphors or metaphoric mapping (see Landau et al. 2010). Metaphoric mapping occurs when, for instance, emotions are mapped onto spatial locations (as described above). But less is known about how other abstract concepts, such as time, are mapped onto space. Xue et al. use event-related potentials from Chinese-English bilinguals to demonstrate that sensorimotor systems 
activate during the processing of temporal markers embedded in sentences presented in these two languages. Although the authors refer to embodied theories of cognition, they present interesting ideas concerning how metaphoric mappings can explain the results that were found.

Behavioural and neuropsychological evidence indicates that language is grounded in the brain's motor system (see current evidence by Cardona et al. 2014). Although most studies in the embodiment framework have used emotion, action and abstract words, very few studies have focused on concepts that refer to schematic representations of embodied experiences. Prieto Velasco and Tercedor Sánchez investigate how concepts referring to image schemas underlie verbal and visual representations of the human body in medical texts. The authors focus particularly on the concept of 'pain'. Their work provides insights as to how the concept of 'pain' is verbally and graphically represented from a medical standpoint. Their findings are in line with current research in cognitive linguistics suggesting that conceptual knowledge, via words and concepts, is closely linked to perceptual properties (see Milin and Zdravković 2013).

Embodied cognition has evolved from the biological notion of autopoiesis (see Maturana 2002) to the psychological notion of embodiment which is now enriched through its application in accounting for social cognition. Such evolution from biological to psychological notions is evidenced by recent research demonstrating that from early developmental stages, humans display bodily interactions that can be seen as precursors of social skills (see interesting work by Castiello et al. 2010). Thus, EmSoCo is a new field of research that extends the broad field of cognitive science. It is expected that future work will not only address new and compelling questions but will also develop new methodologies to help answer these questions. We hope the articles presented in the current special section will provide novel insights on the ways in which social processes reflect the embodiment of cognition. While EmSoCo is still in its infancy, the present studies form a basis for future studies that will either corroborate, extend or even refute the present findings.

\section{References}

Ansorge U, Bohner G (2013) Investigating the association between valence and elevation with an implicit association task that requires upward and downward responding. Univ Psychol 12(5):1453-1471

Cardona JF, Kargieman L, Sinay V, Gershanik O, Gelormini C, Amoruso L, Roca M, Pineda D, Trujillo N, Michon M, García A, Szenkman D, Bekinschtein T, Manes F, Ibáñez A (2014) How embodied is action language? Neurological evidence from motor diseases. Cognition 131(2):311-322

Castiello U, Becchio C, Zoia S, Nelini C, Sartori L, Blason L, D’Ottavio G, Bulgheroni M, Gallese V (2010) Wired to be social: the ontogeny of human interaction. PLoS One 5(10):e13199. doi:10.1371/journal.pone.0013199

Cevasco J, Marmolejo-Ramos F (2013) The importance of studying the role of prosody in the comprehension of spontaneous spoken discourse. Rev Latinoam Psicol 45(1):21-33

Eerland A, Guadalupe T, Zwaan RA (2012) Posture as index for approach-avoidance behavior. PLoS One 7(2):e31291. doi:10. 1371/journal.pone.0031291

Landau MJ, Keefer LA, Meier BP (2010) A metaphor-enriched social cognition. Psychol Bull 136(6):1046-1067

Leung A, Qiu L, Ong L, Tam K-P (2011) Embodied cultural cognition: situating the study of embodied cognition in sociocultural contexts. Soc Pers Psychol Compass 5(9):591-608

Maturana H (2002) Autopoiesis, structural coupling and cognition: a history of these and other notions in the biology of cognition. Cybern Hum Knowing 9(3-4):5-34

Milin P, Zdravković S (2013) Bi-dimensional semantic scales: the embodied maps of meanings. Univ Psychol 12(5):1543-1558

Tagai K, Takata S, Nagai M, Watanabe K, Kumada T (2013) A nearinfrared spectroscopy study of differential brain responses to one or two-handed handing actions: an implication for cultural difference in perceived politeness. Univ Psychol 12(5): $1567-1581$ 